Bull. Austral. Math. Soc.

VOL. $71(2005)$ [81-105]

\title{
WORST-CASE ERRORS IN A SOBOLEV SPACE SETTING FOR CUBATURE OVER THE SPHERE $S^{2}$
}

\author{
Kerstin Hesse and Ian H. Sloan
}

This paper studies the problem of numerical integration over the unit sphere $S^{2} \subseteq \mathbb{R}^{3}$ for functions in the Sobolev space $H^{3 / 2}\left(S^{2}\right)$. We consider sequences $Q_{m(n)}, n \in \mathbb{N}$, of cubature (or numerical integration) rules, where $Q_{m(n)}$ is assumed to integrate exactly all (spherical) polynomials of degree $\leqslant n$, and to use $m=m(n)$ values of $f$. The cubature weights of all rules $Q_{m(n)}$ are assumed to be positive, or alternatively the sequence $Q_{m(n)}, n \in \mathbb{N}$, is assumed to have a certain local regularity property which involves the weights and the points of the rules $Q_{m(n)}, n \in \mathbb{N}$. Under these conditions it is shown that the worst-case (cubature) error, denoted by $E_{3 / 2}\left(Q_{m(n)}\right)$, for all functions in the unit ball of the Hilbert space $H^{3 / 2}\left(S^{2}\right)$ satisfies the estimate $E_{3 / 2}\left(Q_{m(n)}\right) \leqslant c n^{-3 / 2}$, where the constant $c$ is a universal constant for all sequences of positive weight cubature rules. For a sequence $Q_{m(n)}, n \in \mathbb{N}$, of cubature rules that satisfies the alternative local regularity property the constant $c$ may depend on the sequence $Q_{m(n)}, n \in \mathbb{N}$. Examples of cubature rules that satisfy the assumptions are discussed.

\section{INTRODUCTION}

In this paper we study the problem of numerical integration over the unit sphere $S^{2} \subseteq \mathbb{R}^{3}$

$$
S^{2}=\left\{\mathbf{x}=(x, y, z) \in \mathbb{R}^{3} \mid x^{2}+y^{2}+z^{2}=1\right\} .
$$

The exact integral of a (continuous) function $f$ defined on $S^{2}$ is

$$
I f:=\int_{S^{2}} f(\mathbf{x}) d \omega(\mathbf{x})
$$

where $d \omega(\mathbf{x})$ denotes the surface measure on $S^{2}$. In terms of the usual polar coordinates $\theta, \phi$, the integral takes the explicit form

$$
I f=\int_{0}^{2 \pi} \int_{0}^{\pi} f(\sin \theta \cos \phi, \sin \theta \sin \phi, \cos \theta) \sin \theta d \theta d \phi .
$$

Received 25th August, 2004

The support of the Australian Research Council under its Centre of Excellence program is gratefully acknowledged.

Copyright Clearance Centre, Inc. Serial-fee code: 0004-9727/05 \$A2.00+0.00. 
We are interested in the approximation of $I f$ by cubature rules of the form

$$
Q_{m} f:=\sum_{j=1}^{m} w_{j} f\left(\mathbf{x}_{j}\right),
$$

where

$$
\mathbf{x}_{j} \in S^{2}, \quad w_{j} \in \mathbb{R}, \quad j=1, \ldots, m .
$$

Most of the time we shall assume also that $Q_{m}$ integrates exactly all (spherical) polynomials of degree $\leqslant n$. Thus we assume $m=m(n)$, and

$$
Q_{m} p=I p \quad \forall p \in \mathbb{P}_{n},
$$

where $\mathbb{P}_{n}$ is the set of spherical polynomials of degree $\leqslant n$, that is the set of all polynomials in $x, y, z$ of total degree at most $n$, restricted to the unit sphere $S^{2}$. It is important for our considerations that $\operatorname{dim} \mathbb{P}_{n}=(n+1)^{2}$.

There are many cubature rules with the property (1.4). Most obviously, there are product Gauss rules discussed by Stroud [18], considered in detail in Section 6 . But there are also many others (see Section 6).

The integrands $f$ are assumed to be selected from a particular Sobolev space $H^{s}$ $=H^{s}\left(S^{2}\right)$, with $s>1$. (Roughly, $H^{s}$ is the space of square-integrable functions on $S^{2}$ whose generalised (distributional) derivatives of order $s$ are also square-integrable. Precise definitions are given in Section 2.)

By definition, the worst-case error for the cubature rule $Q_{m}$ in the space $H^{s}$, endowed with the norm $\|\cdot\|_{s}$, is

$$
E_{s}\left(Q_{m}\right):=\sup _{\substack{f \in H^{\circ},\|f\|_{s} \leqslant 1}}\left|Q_{m} f-I f\right| .
$$

For a particular choice of $s>1$, and a well chosen sequence $\left(Q_{m(n)}\right)_{n \in \mathbb{N}}$ of cubature rules, how fast can $E_{s}\left(Q_{m(n)}\right)$ converge to zero as $n \rightarrow \infty$ ? The present paper is motivated by persuasive numerical evidence, presented in [15], suggesting that, for the particular case of $s=3 / 2$ and for cubature rules based on extremal systems,

$$
E_{3 / 2}\left(Q_{m(n)}\right) \leqslant c(m(n))^{-3 / 4}=c(n+1)^{-3 / 2} .
$$

An extremal system is a set of $(n+1)^{2}$ points for which the determinant of the interpolation matrix with respect to any fixed basis of $\mathbb{P}_{n}$ takes its greatest possible value. The cubature rules in [15] are obtained by exact integration of the interpolating spherical polynomial in $\mathbb{P}_{n}$ that coincides with a given function at the $(n+1)^{2}$ points of an extremal system. The analysis in [15] obtains only the much weaker result $E_{3 / 2}\left(Q_{m(n)}\right) \leqslant c n^{-1 / 2}$. (In this paper $c$ denotes a generic constant, which may take different values in different places, while $c_{0}, c_{1}, \ldots$ denote constants with fixed values.) 
In this paper we do indeed prove that

$$
E_{3 / 2}\left(Q_{m(n)}\right) \leqslant c n^{-3 / 2},
$$

under the assumption that, for each $n \in \mathbb{N}, Q_{m(n)}$ satisfies $Q_{m(n)} p=I p$ for all $p \in \mathbb{P}_{n}$ and that the weights $w_{j}$ of $Q_{m(n)}$ are positive. The constant $c$ in (1.6) is in this case independent of the particular sequence $\left(Q_{m(n)}\right)$ of positive weight cubature rules. The assumption of positive weights can be replaced by the assumption that $\left(Q_{m(n)}\right)$ satisfies a certain local regularity property, in which case the constant $c$ in (1.6) may depend on the sequence of cubature rules $\left(Q_{m(n)}\right)$. (The precise statement is given in Theorem 6.) Examples of cubature rules satisfying the conditions are discussed in Section 6. For the case of the rules corresponding to the extremal systems in [15] the reported weights, for degree $n$ up to 50, are all positive.

The proof of Theorem 6 rests on an unusual representation (in Lemma 9) of the tail of a certain Legendre series as the sum of a polynomial and other terms involving a more rapidly converging Legendre series. The proof of Lemma 9 (in Section 5) uses the Christoffel-Darboux formula to give an integral representation of the tail of a particular Legendre series, and the integrand in this integral representation is then manipulated algebraically. The proof of Lemma 9 does not extend in an obvious way to general values of $s>1$.

\section{PRELIMINARIES}

Let $L_{2}=L_{2}\left(S^{2}\right)$ be the space of square-integrable measurable functions on $S^{2}$, that is, the set of measurable functions $f$ on $S^{2}$ for which

$$
\int_{S^{2}}|f(\mathbf{x})|^{2} d \omega(\mathbf{x})<\infty .
$$

The space $L_{2}$ is a Hilbert space with the inner product

$$
(f, g)_{L_{2}}:=\int_{S^{2}} f(\mathbf{x}) g(\mathbf{x}) d \omega(\mathbf{x}), \quad f, g \in L_{2} .
$$

On the unit sphere $S^{2} \subseteq \mathbb{R}^{3}$, for $\ell \in \mathbb{N}_{0}$ let

$$
\left\{Y_{\ell k} \mid k=1, \ldots, 2 \ell+1\right\}
$$

be an orthonormal set (with respect to $(\cdot, \cdot)_{L_{2}}$ ) of (real) spherical harmonics of exact degree $\ell$. Of central importance is the addition theorem of spherical harmonics (see [9]),

$$
\sum_{k=1}^{2 \ell+1} Y_{\ell k}(\mathbf{x}) Y_{\ell k}(\mathbf{y})=\frac{2 \ell+1}{4 \pi} P_{\ell}(\mathbf{x} \cdot \mathbf{y}), \quad \mathbf{x}, \mathbf{y} \in S^{2}
$$


where $\mathbf{x} \cdot \mathbf{y}$ denotes the usual Euclidean inner product of $\mathbf{x}$ and $\mathbf{y}$ in $\mathbb{R}^{3}$, and $P_{\ell}$ is the Legendre polynomial of degree $\ell$.

A function $f \in L_{2}$ is equal in the $L_{2}$-sense to its Laplace (or Fourier) series,

$$
f(\mathbf{x}) \sim \sum_{\ell=0}^{\infty} \sum_{k=1}^{2 \ell+1} \widehat{f}_{\ell k} Y_{\ell k}(\mathbf{x})
$$

where

$$
\widehat{f}_{\ell k}:=\int_{S^{2}} f(\mathbf{x}) Y_{\ell k}(\mathbf{x}) d \omega(\mathbf{x})
$$

For $s \geqslant 0$, the Sobolev space $H^{s} \subseteq L^{2}$ may be defined as the completion of the space of all spherical polynomials, $\bigcup_{\ell=0}^{\infty} \mathbb{P}_{\ell}$, with respect to the norm

$$
\|f\|_{s}:=\left(\sum_{\ell=0}^{\infty} \sum_{k=1}^{2 \ell+1}\left(\ell+\frac{1}{2}\right)^{2 s} \hat{f}_{\ell k}^{2}\right)^{1 / 2} .
$$

(We follow [4] in the definition of the norm.) The space $H^{s}$ is a Hilbert space, with inner product

$$
(f, g)_{s}:=\sum_{\ell=0}^{\infty} \sum_{k=1}^{2 \ell+1}\left(\ell+\frac{1}{2}\right)^{2 s} \widehat{f}_{\ell k} \widehat{g}_{\ell k}, \quad f, g \in H^{s} .
$$

For $s>1$ it follows from the Cauchy-Schwarz inequality that

$$
\begin{aligned}
\left|\sum_{\ell=N}^{\infty} \sum_{k=1}^{2 \ell+1} \widehat{f}_{\ell k} Y_{\ell k}(\mathbf{x})\right| & =\left|\sum_{\ell=N}^{\infty} \sum_{k=1}^{2 \ell+1}\left(\ell+\frac{1}{2}\right)^{s} \widehat{f}_{\ell k}\left(\ell+\frac{1}{2}\right)^{-s} Y_{\ell k}(\mathbf{x})\right| \\
& \leqslant\|f\|_{s}\left(\sum_{\ell=N}^{\infty} \sum_{k=1}^{2 \ell+1}\left(\ell+\frac{1}{2}\right)^{-2 s} Y_{\ell k}^{2}(\mathbf{x})\right)^{1 / 2} \\
& =C_{s, N}\|f\|_{s}
\end{aligned}
$$

where with the help of the addition theorem (2.1)

$$
C_{s, N}:=\left(\frac{1}{2 \pi} \sum_{\ell=N}^{\infty}\left(\ell+\frac{1}{2}\right)^{1-2 s} P_{\ell}(1)\right)^{1 / 2}=\left(\frac{1}{2 \pi} \sum_{\ell=N}^{\infty}\left(\ell+\frac{1}{2}\right)^{1-2 s}\right)^{1 / 2},
$$

which is finite for all $N \in \mathbb{N}_{0}$ and converges to zero as $N \rightarrow \infty$, because $1-2 s<-1$. Thus the Fourier series of $f \in H^{s}$, for $s>1$, converges uniformly, and hence converges to a continuous function that coincides almost everywhere with $f$. Taking $f$ as the uniform limit of the Fourier series, it follows that $f$ is continuous, and (on setting $N=0$ in (2.3))

$$
|f(\mathbf{x})|=\left|\sum_{\ell=0}^{\infty} \sum_{k=1}^{2 \ell+1} \widehat{f}_{\ell k} Y_{\ell k}(\mathbf{x})\right| \leqslant C_{s, 0}\|f\|_{s} \quad \forall \mathbf{x} \in S^{2} .
$$


Hence

$$
\sup _{\mathbf{x} \in S^{2}}|f(\mathbf{x})| \leqslant C_{s, 0}\|f\|_{s},
$$

and we obtain an embedding of $H^{s}$ into $C\left(S^{2}\right)$. The estimate (2.4) also implies that for $s>1$ point evaluation is a bounded linear functional on $H^{s}$, and hence $H^{s}$ is a reproducing kernel Hilbert space. That is to say, there exists $K_{s}: S^{2} \times S^{2} \rightarrow \mathbb{R}$, with $K_{s}(\mathbf{x}, \mathbf{y})=K_{s}(\mathbf{y}, \mathbf{x})$ and $K_{s}(\mathbf{x}, \cdot) \in H^{s}$ for all $\mathbf{x}, \mathbf{y} \in S^{2}$, such that

$$
\left(f, K_{s}(\cdot, \mathbf{y})\right)_{s}=f(\mathbf{y}) \quad \forall f \in H^{s}, \forall \mathbf{y} \in S^{2} .
$$

From (2.5), it can be easily seen that the reproducing kernel $K_{s}(\mathbf{x}, \mathbf{y})$ is given explicitly by

$$
\begin{aligned}
K_{s}(\mathbf{x}, \mathbf{y}) & =\sum_{\ell=0}^{\infty} \sum_{k=1}^{2 \ell+1}\left(\ell+\frac{1}{2}\right)^{-2 s} Y_{\ell k}(\mathbf{x}) Y_{\ell k}(\mathbf{y}) \\
& =\frac{1}{2 \pi} \sum_{\ell=0}^{\infty}\left(\ell+\frac{1}{2}\right)^{1-2 s} P_{\ell}(\mathbf{x} \cdot \mathbf{y})
\end{aligned}
$$

which converges absolutely and uniformly for $(\mathbf{x}, \mathbf{y}) \in S^{2} \times S^{2}$ because $s>1$. Thus $K_{s}$ is a continuous function on $S^{2} \times S^{2}$.

\section{WORST-CASE CUBATURE ERROR IN A REPRODUCING KERNEL HILbERT SPACE}

Let $H$ be a reproducing kernel Hilbert space of real continuous functions defined on $S^{2}$, with inner product $(\cdot, \cdot)$, norm $\|\cdot\|$, and reproducing kernel $K$. We also assume that $H$ can be embedded into $C\left(S^{2}\right)$, that is, there exists a constant $c$ such that $\sup _{\mathbf{x} \in S^{2}}|f(\mathbf{x})|$ $\leqslant c\|f\|$ for all $f \in H$. For $f$ in the space $H$ let

$$
Q_{m} f:=\sum_{j=1}^{m} w_{j} f\left(\mathbf{x}_{j}\right)
$$

be an $m$-point cubature formula. (Of course $Q_{m} f$ is well defined, because point evaluation is well defined for $f \in H$.) We assume that $Q_{m} f$ is an approximation to $I f$, the integral of $f$ over $S^{2}$,

$$
\text { If }:=\int_{S^{2}} f(\mathbf{x}) d \omega(\mathbf{x}) .
$$

Since $H$ is a reproducing kernel Hilbert space with reproducing kernel $K$, we can write

$$
f\left(\mathbf{x}_{j}\right)=\left(f, K\left(\cdot, \mathbf{x}_{j}\right)\right)
$$

and hence

$$
Q_{m} f=\left(f, \sum_{j=1}^{m} w_{j} K\left(\cdot, \mathbf{x}_{j}\right)\right) .
$$


Similarly, integration over $S^{2}$ is a bounded linear functional on $H$ with representer $\int_{S^{2}} K(\cdot, \mathbf{y}) d \omega(\mathbf{y})$, that is

$$
I f=\left(f, \int_{S^{2}} K(\cdot, \mathbf{y}) d \omega(\mathbf{y})\right)
$$

Thus the cubature error is

$$
Q_{m} f-I f=\left(f, \sum_{j=1}^{m} w_{j} K\left(\cdot, \mathbf{x}_{j}\right)-\int_{S^{2}} K(\cdot, \mathbf{y}) d \omega(\mathbf{y})\right)
$$

The worst-case (cubature) error for the cubature rule $Q_{m}$ in the space $H$ is defined as

$$
E\left(Q_{m}\right):=\sup _{\substack{f \in H,\|f\| \leqslant 1}}\left|Q_{m} f-I f\right| .
$$

From (3.1) it follows easily that

$$
E\left(Q_{m}\right)=\left\|\sum_{j=1}^{m} w_{j} K\left(\cdot, \mathbf{x}_{j}\right)-\int_{S^{2}} K(\cdot, \mathbf{y}) d \omega(\mathbf{y})\right\|
$$

In turn, on using $\|g\|=(g, g)^{1 / 2}$, together with the reproducing property $(2.5)$ of $K$, it follows that

$$
\begin{aligned}
E\left(Q_{m}\right)=\left(\sum_{i=1}^{m} \sum_{j=1}^{m} w_{i} w_{j} K\left(\mathbf{x}_{i}, \mathbf{x}_{j}\right)-2 \sum_{j=1}^{m} w_{j} \int_{S^{2}} K\left(\mathbf{x}_{j}, \mathbf{y}\right) d \omega(\mathbf{y})\right. & \\
& \left.\quad+\int_{S^{2}} \int_{S^{2}} K(\mathbf{z}, \mathbf{y}) d \omega(\mathbf{z}) d \omega(\mathbf{y})\right)^{\mathbf{1} / 2}
\end{aligned}
$$

So far our reproducing kernel Hilbert space is rather general. The expression (3.2) is in fact well known (see, for example [16]), and has been found useful in other settings.

\section{WORST-CASE CUBATURE ERROR IN $H^{s}$}

Now we specialise our discussion of worst-case cubature error to the particular case of the Hilbert space $H^{s}$, with $s>1$, for which the reproducing kernel $K_{s}$ is given by (2.6). Moreover, let us assume that the weights of the cubature rule satisfy

$$
\sum_{j=1}^{m} w_{j}=4 \pi=\int_{S^{2}} d \omega(\mathbf{x})
$$

so that the cubature rule is exact at least for constant functions. It follows immediately from (2.6) (on noticing that in the series representation (2.6) of $K_{s}$ the terms with $\ell \neq 0$ 
contribute nothing to the integral) that

$$
\int_{S^{2}} K_{s}(\mathbf{x}, \mathbf{y}) d \omega(\mathbf{y})=2^{2 s} \quad \forall \mathbf{x} \in S^{2} .
$$

This allows the second and third terms of (3.2) to be evaluated explicitly, yielding the simplified expression

$$
E_{s}\left(Q_{m}\right)=\left(\sum_{i=1}^{m} \sum_{j=1}^{m} w_{i} w_{j} K_{s}^{(1)}\left(\mathbf{x}_{i}, \mathbf{x}_{j}\right)\right)^{1 / 2},
$$

where $K_{s}^{(a)}, a \in \mathbb{N}$, denotes the contribution to the reproducing kernel (2.6) from the terms with $\ell \geqslant a$; that is,

$$
K_{s}^{(a)}(\mathbf{x}, \mathbf{y})=\frac{1}{2 \pi} \sum_{\ell=a}^{\infty}\left(\ell+\frac{1}{2}\right)^{1-2 s} P_{\ell}(\mathbf{x} \cdot \mathbf{y})
$$

We want to study the asymptotic behaviour of the worst-case error for a sequence of cubature rules $\left(Q_{m(n)}\right)$, where $Q_{m(n)}$ is exact for all polynomials of degree $\leqslant n$, that is, (1.4) is satisfied. From this it follows that for $1 \leqslant \ell \leqslant n$ we have

$$
\sum_{j=1}^{m} w_{j} P_{\ell}\left(\mathbf{x} \cdot \mathbf{x}_{j}\right)=\int_{S^{2}} P_{\ell}(\mathbf{x} \cdot \mathbf{y}) d \omega(\mathbf{y})=0 \quad \forall \mathbf{x} \in S^{2},
$$

leading to (4.2) being further simplified to

$$
E_{s}\left(Q_{m(n)}\right)=\left(\sum_{i=1}^{m} \sum_{j=1}^{m} w_{i} w_{j} K_{s}^{(n+1)}\left(\mathbf{x}_{i}, \mathbf{x}_{j}\right)\right)^{1 / 2} .
$$

The main result of this paper, Theorem 6 below, is restricted to the case $s=3 / 2$, and also requires that the sequence of cubature rules has a certain regularity property expressed in terms of spherical caps.

Definition 1: For each point $\mathbf{x} \in S^{2}$, let $S(\mathbf{x}, r)$ be the 'spherical cap' with axis $\mathbf{X}$ and angular radius $r$, that is

$$
S(\mathbf{x}, r):=\left\{\mathbf{y} \in S^{2} \mid \cos ^{-1}(\mathbf{x} \cdot \mathbf{y}) \leqslant r\right\} .
$$

The surface measure (or area) of $S(\mathbf{x}, r)$ is denoted by $|S(\mathbf{x}, r)|$.

Lemma 2. Let $Q_{m}$ be a cubature rule on $S^{2}$, with points $\mathrm{x}_{j} \in S^{2}$ and weights $w_{j} \in \mathbb{R}, j=1, \ldots, m$. Assume that there exist $r_{0} \in(0, \pi]$ and a constant $c_{0}>0$ such that for all $\mathrm{x} \in S^{2}$

$$
\sum_{\substack{j=1, \mathbf{x}_{j} \in S\left(\mathbf{x}, r_{0}\right)}}^{m}\left|w_{j}\right| \leqslant c_{0}\left|S\left(\mathbf{x}, r_{0}\right)\right|
$$


Then for all $r \in\left[r_{0}, \pi\right]$ and for all $\mathbf{x} \in S^{2}$

$$
\sum_{\substack{j=1, \mathbf{x}_{j} \in S(\mathbf{x}, r)}}^{m}\left|w_{j}\right| \leqslant\left(1+\frac{3 \pi^{2}}{4}\right) c_{0}|S(\mathbf{x}, r)| .
$$

In particular, (4.6) implies that

$$
\sum_{j=1}^{m}\left|w_{j}\right| \leqslant 4 \pi\left(1+\frac{3 \pi^{2}}{4}\right) c_{0} .
$$

The proof of Lemma 2 will be given in Section 5 .

COROLlary 3. Let the assumptions be the same as in Lemma 2, with $r_{0} \in(0,(\pi / 2)]$. Then for every spherical cap $S(\mathbf{x}, r)$ of angular radius $r_{0} \leqslant r \leqslant \pi / 2$

$$
\sum_{\substack{j=1, \mathbf{x}_{j} \in \mathcal{S}(\mathbf{x}, r)}}^{m}\left|w_{j}\right| \leqslant 2 \pi\left(1+\frac{3 \pi^{2}}{4}\right) c_{0}(\sin r)^{2} .
$$

Proof: The area of $S(\mathbf{x}, r)$ is given by

$$
|S(\mathbf{x}, r)|=2 \pi(1-\cos r) .
$$

Observing that $1-\cos r=(\sin r)^{2}(1+\cos r)^{-1}$, and that for $r \leqslant \pi / 2$ we have $(1+\cos r)^{-1} \leqslant 1$, we obtain (4.8) from (4.6).

Definition 4: (Property $(\mathrm{R})$ ) A sequence of cubature rules $\left(Q_{m(n)}\right)$ is said to have the property $(R)$, or to be 'quadrature regular' (see [14]), if there exist positive constants $c_{0}$ and $c_{1}$ independent of $n$ with $c_{1} \leqslant \pi / 2$, such that for all $n \geqslant 1$ the points $\mathbf{x}_{j}$ and the weights $w_{j}, j=1, \ldots, m$, of $Q_{m(n)}$ satisfy

$$
\sum_{\substack{j=1, \mathbf{x}_{j} \in S\left(\mathbf{x},\left(c_{1} / n\right)\right)}}^{m}\left|w_{j}\right| \leqslant c_{0}\left|S\left(\mathbf{x}, \frac{c_{1}}{n}\right)\right| \quad \forall \mathbf{x} \in S^{2} .
$$

REMARK 5. The assumption $c_{1} \leqslant \pi / 2$ in the property (R) can be removed, because (4.9) with a constant $c_{1}>\pi / 2$ implies after a change of the constant $c_{0}$ that (4.9) is also satisfied with a new constant $c_{1} \leqslant \pi / 2$. Furthermore it is sufficient if (4.9) is satisfied for all $n \geqslant N$, because with a change of $c_{0}$ we can arrange that (4.9) is satisfied for all $n \geqslant 1$.

If the property (R) holds, then for each $n \in \mathbb{N}$ the cubature rule $Q_{m(n)}$ satisfies the hypothesis of Lemma 2 with $r_{0}=c_{1} / n$. We make use of this fact in the proof of the following theorem, which is the main result of the paper.

TheOREM 6. Assume that $\left(Q_{m(n)}\right)$ is an infinite sequence of cubature rules on $S^{2}$, where $Q_{m(n)}$ is an $m(n)$-point rule satisfying $Q_{m(n)} p=I p$ for all $p \in \mathbb{P}_{n}$. Assume also that the sequence $\left(Q_{m(n)}\right)$ has the property $(R)$. Then there exists $c>0$ such that

$$
E_{3 / 2}\left(Q_{m(n)}\right) \leqslant c n^{-3 / 2} \quad \forall n \geqslant 1 .
$$


REMARK 7. The constant $c$ in (4.10) in Theorem 6 depends on the constants $c_{0}$ and $c_{1}$ in the regularity property (R), and therefore may depend on the sequence $\left(Q_{m(n)}\right)$ of cubature rules. However, Reimer [12, Lemma 1] shows that if the weights are all positive then (4.9) holds automatically, with universal constants $c_{0}$ and $c_{1}$, under the assumption that $Q_{m(n)} p=I p$ for all $p \in \mathbb{P}_{n}$. Thus the constants $c_{0}$ and $c_{1}$ in (4.9) are in this case independent of $\left(Q_{m(n)}\right)$, and so also is the constant $c$ in (4.10). We formulate this stronger result as a corollary.

COROLLARY 8. There exists a constant $c>0$ such that for any positive weight $m(n)$-point cubature rule $Q_{m(n)}$, with $Q_{m(n)} p=I p$ for all $p \in \mathbb{P}_{n}$, the worst-case cubature error in $H^{3 / 2}$ satisfies

$$
E_{3 / 2}\left(Q_{m(n)}\right) \leqslant c n^{-3 / 2}
$$

The proof of Theorem 6 will proceed by way of two lemmas and a corollary.

In the following $P_{\ell}^{(\alpha, \beta)}$ denotes the Jacobi polynomial of degree $\ell$ and indices $\alpha, \beta>-1$, as defined by [19, Chapter II, 2.4, and Chapter IV], thus satisfying the orthogonality relation

$$
\int_{-1}^{1} P_{\ell}^{(\alpha, \beta)}(t) P_{m}^{(\alpha, \beta)}(t)(1-t)^{\alpha}(1+t)^{\beta} d t=0 \quad \forall m, \ell \in \mathbb{N}_{0} \text { with } \ell \neq m .
$$

The particular Jacobi polynomial $P_{\ell}^{(1,0)}$ assumes its maximum at $t=1$, more precisely $\left|P_{\ell}^{(1,0)}(t)\right| \leqslant P_{\ell}^{(1,0)}(1)=(\ell+1)$ for all $t \in[-1,1]$, while $P_{\ell}^{(1,0)}(-1)=(-1)^{\ell}$.

The first lemma gives a representation of

$$
K_{3 / 2}^{(n+1)}(\mathbf{x}, \mathbf{y})=\frac{1}{2 \pi} \sum_{\ell=n+1}^{\infty}\left(\ell+\frac{1}{2}\right)^{-2} P_{\ell}(\mathbf{x} \cdot \mathbf{y})
$$

in a convenient form for further analysis.

Lemma 9. For $n \geqslant 0$ and $-1 \leqslant t<1$,

$$
\begin{aligned}
\frac{1}{2 \pi} \sum_{\ell=n+1}^{\infty}\left(\ell+\frac{1}{2}\right)^{-2} P_{\ell}(t)=- & \frac{2}{\pi} \frac{(n+1)}{(2 n-1)(2 n+1)(2 n+3)} P_{n}^{(1,0)}(t) \\
& +\frac{12}{\pi} \frac{(n+1)}{(2 n-1)(2 n+1)(2 n+3)(2 n+5)} \frac{P_{n}(t)}{1-t} \\
& -\frac{36}{\pi} \frac{1}{1-t} \sum_{\ell=n+1}^{\infty} \frac{1}{(2 \ell-3)(2 \ell-1)(2 \ell+3)(2 \ell+5)} P_{\ell}(t) \\
& -\frac{8}{\pi} \sum_{\ell=n+1}^{\infty} \frac{1}{(2 \ell-1)(2 \ell+1)^{2}(2 \ell+3)} P_{\ell}(t) .
\end{aligned}
$$

Corollary 10. For $\mathbf{x}, \mathbf{y} \in S^{2}$ and $\mathbf{x} \cdot \mathbf{y}=\cos \theta$, with $0<\theta<\pi$, there exists a constant $c>0$, independent of $n$, such that

$$
\left|K_{3 / 2}^{(n+1)}(\mathbf{x}, \mathbf{y})+\frac{2}{\pi} \frac{(n+1)}{(2 n-1)(2 n+1)(2 n+3)} P_{n}^{(1,0)}(\cos \theta)\right| \leqslant c n^{-7 / 2}(\sin \theta)^{-5 / 2}
$$


for all $n \in \mathbb{N}_{0}$, and hence for fixed $\mathbf{x}, \mathbf{y}$

$$
K_{3 / 2}^{(n+1)}(\mathbf{x}, \mathbf{y})=n^{-5 / 2} D_{n}(\theta)+O\left(n^{-7 / 2}\right)
$$

where

$$
\underset{n \rightarrow \infty}{\limsup }\left|D_{n}(\theta)\right|>0 \text {. }
$$

REMARK 11. The corollary brings to attention a delicate aspect of the proof of Theorem 6. To prove the theorem we must show that (from (4.4))

$$
E_{3 / 2}^{2}\left(Q_{m(n)}\right)=\sum_{i=1}^{m} \sum_{j=1}^{m} w_{i} w_{j} K_{3 / 2}^{(n+1)}\left(\mathbf{x}_{i}, \mathbf{x}_{j}\right)
$$

is of order $O\left(n^{-3}\right)$. Yet for fixed $\mathbf{x}, \mathbf{y}$, with $\mathbf{x} \neq \mathbf{y}$ and $\mathbf{x} \neq-\mathbf{y}$, the second conclusion in the corollary tells us that for large $n$ the kernel $K_{3 / 2}^{(n+1)}(\mathbf{x} \cdot \mathbf{y})$ is larger than the desired $O\left(n^{-3}\right)$ result for (4.15). The troublesome term is, of course, the first term on the righthand side of (4.12). Fortunately, the order of this term is improved (by an additional factor of $n^{-1 / 2}$ ) through the application of the cubature sum, as the following simple lemma shows. The key is that, because $P_{n}^{(1,0)}$ is a polynomial of degree $n$, we can make yet another application of the exactness property (1.4) of the cubature sum.

LEMMA 12. For $n \geqslant 1$, assume that $Q_{m}$ is an m-point cubature rule on $S^{2}$ satisfying $Q_{m} p=I p$ for all $p \in \mathbb{P}_{n}$. Then

$$
\sum_{i=1}^{m} \sum_{j=1}^{m} w_{i} w_{j} P_{n}^{(1,0)}\left(\mathbf{x}_{i} \cdot \mathbf{x}_{j}\right)=\frac{16 \pi^{2}}{n+1}
$$

Lemma 9, Corollary 10, and Lemma 12 will be proved in Section 5.

PROOF OF THEOREM 6: In order to deal separately with the first term on the right-hand side of (4.12), we rewrite (4.15), using (4.11) and (4.12), as

$$
\begin{aligned}
E_{3 / 2}^{2}\left(Q_{m(n)}\right)=\sum_{i=1}^{m} \sum_{j=1}^{m} w_{i} w_{j}\left(-\frac{2}{\pi}\right) \frac{(n+1)}{(2 n-1)(2 n+1)(2 n+3)} & P_{n}^{(1,0)}\left(\mathbf{x}_{i} \cdot \mathbf{x}_{j}\right) \\
& +\sum_{i=1}^{m} \sum_{j=1}^{m} w_{i} w_{j} k_{n}\left(\mathbf{x}_{i}, \mathbf{x}_{j}\right)
\end{aligned}
$$

where for $\mathbf{x}, \mathbf{y} \in S^{2}$

$$
k_{n}(\mathbf{x}, \mathbf{y}):=K_{3 / 2}^{(n+1)}(\mathbf{x}, \mathbf{y})+\frac{2}{\pi} \frac{(n+1)}{(2 n-1)(2 n+1)(2 n+3)} P_{n}^{(1,0)}(\mathbf{x} \cdot \mathbf{y})
$$

Applying Lemma 12, we obtain

$$
E_{3 / 2}^{2}\left(Q_{m(n)}\right)=-\frac{32 \pi}{(2 n-1)(2 n+1)(2 n+3)}+\sum_{i=1}^{m} \sum_{j=1}^{m} w_{i} w_{j} k_{n}\left(\mathbf{x}_{i}, \mathbf{x}_{j}\right)
$$


Clearly, the first term is of the desired order $O\left(n^{-3}\right)$. We split the remaining term, writing

$$
E_{3 / 2}^{2}\left(Q_{m(n)}\right)=O\left(n^{-3}\right)+A^{+}+A^{-}
$$

where

$$
A^{ \pm}:=\sum_{i=1}^{m} w_{i} \sum_{\substack{j=1, \mathbf{x}_{j} \in H_{i}^{ \pm}}}^{m} w_{j} k_{n}\left(\mathbf{x}_{i}, \mathbf{x}_{j}\right),
$$

and where for $i=1, \ldots, m$

$$
\begin{aligned}
& H_{i}^{+}:=\left\{\mathbf{x} \in S^{2} \mid \mathbf{x}_{i} \cdot \mathbf{x} \geqslant 0\right\}, \\
& H_{i}^{-}:=S^{2} \backslash H_{i}^{+} .
\end{aligned}
$$

That is, with respect to $\mathbf{x}_{i}$ as the 'north pole' we decompose the set $S^{2}$ into a 'northern' hemisphere $H_{i}^{+}$and a 'southern' hemisphere $H_{i}^{-}$, arbitrarily putting points on the equator in $H_{i}^{+}$.

The estimate (4.13) in Corollary 10 tells us that for $\mathbf{x}, \mathbf{y} \in S^{2}$ and $\mathbf{x} \cdot \mathbf{y}=\cos \theta$, with $\theta \in(0, \pi)$,

$$
\left|k_{n}(\mathbf{x}, \mathbf{y})\right| \leqslant c n^{-7 / 2}(\sin \theta)^{-5 / 2} .
$$

Since this bound diverges toward $\theta=0$ and $\theta=\pi$, it is convenient to split each of the sums $A^{ \pm}$into a 'diagonal' part $D^{ \pm}$, which includes for each $i \in\{1, \ldots, n\}$ the contributions from points $\mathbf{x}_{j}$ that lie in the spherical cap $S\left( \pm \mathbf{x}_{i},\left(c_{1} / n\right)\right)$ centred at $\pm \mathbf{x}_{i}$, and a remainder $R^{ \pm}$. Here $c_{1}$ is the constant in the property (R). Thus we write

$$
A^{ \pm}=D^{ \pm}+R^{ \pm}
$$

where

$$
\begin{aligned}
& D^{ \pm}:=\sum_{i=1}^{m} w_{i} \sum_{\substack{j=1, \mathbf{x}_{j} \in S\left( \pm \mathbf{x}_{i}, \frac{c_{1}}{n}\right) \cap H_{i}^{ \pm}}}^{m} w_{j} k_{n}\left(\mathbf{x}_{i}, \mathbf{x}_{j}\right), \\
& R^{ \pm}:=\sum_{i=1}^{m} w_{i} \sum_{\substack{j=1, \mathbf{x}_{j} \in H_{i}^{ \pm} \backslash S\left( \pm \mathbf{x}_{i}, c_{\frac{1}{n}}\right)}}^{m} w_{j} k_{n}\left(\mathbf{x}_{i}, \mathbf{x}_{j}\right) .
\end{aligned}
$$

To estimate $D^{ \pm}$we use, from (4.16) and (4.11),

$$
\begin{aligned}
\left|k_{n}(\mathbf{x}, \mathbf{y})\right| & \leqslant \frac{2}{\pi} \frac{(n+1)}{(2 n-1)(2 n+1)(2 n+3)}\left|P_{n}^{(1,0)}(\mathbf{x} \cdot \mathbf{y})\right|+\frac{1}{2 \pi}\left|\sum_{\ell=n+1}^{\infty}\left(\ell+\frac{1}{2}\right)^{-2} P_{\ell}(\mathbf{x} \cdot \mathbf{y})\right| \\
& \leqslant \frac{2}{\pi} \frac{(n+1)^{2}}{(2 n-1)(2 n+1)(2 n+3)}+\frac{1}{2 \pi} \sum_{\ell=n+1}^{\infty}\left(\ell+\frac{1}{2}\right)^{-2} \\
& \leqslant c n^{-1}
\end{aligned}
$$


where we have used $\left|P_{\ell}^{(1,0)}(t)\right| \leqslant(\ell+1)$ and $\left|P_{\ell}(t)\right| \leqslant 1$ for all $t \in[-1,1]$ and all $\ell \geqslant 0$. This yields

$$
\left|D^{ \pm}\right| \leqslant \frac{c}{n} \sum_{i=1}^{m}\left|w_{i}\right| \sum_{\substack{j=1, \mathbf{x}_{j} \in S\left( \pm \mathbf{x}_{i}, \frac{\mathbf{c}_{1}}{n}\right)}}^{m}\left|w_{j}\right|
$$

and hence from the property (R) and (4.7)

$$
\begin{aligned}
\left|D^{ \pm}\right| & \leqslant \frac{c}{n} \sum_{i=1}^{m}\left|w_{i}\right| c_{0}\left|S\left( \pm \mathbf{x}_{i}, \frac{c_{1}}{n}\right)\right| \\
& \leqslant \frac{c c_{0}}{n}\left(\sum_{i=1}^{m}\left|w_{i}\right|\right) \pi c_{1}^{2} n^{-2} \\
& \leqslant \frac{4 \pi^{2} c c_{0} c_{1}^{2} c_{2}}{n^{3}}=\frac{c}{n^{3}}
\end{aligned}
$$

where we have written $c_{2}:=\left(1+\left(3 \pi^{2}\right) / 4\right) c_{0}$, and used

$$
\left|S\left( \pm \mathbf{x}_{i}, \frac{c_{1}}{n}\right)\right|=2 \pi\left(1-\cos \frac{c_{1}}{n}\right)=4 \pi\left(\sin \frac{c_{1}}{2 n}\right)^{2} \leqslant \pi c_{1}^{2} n^{-2} .
$$

It only remains to estimate $R^{ \pm}$. For this purpose we use the estimate for $k_{n}$ from Corollary 10 to obtain

$$
\left|R^{ \pm}\right| \leqslant c n^{-7 / 2} \sum_{i=1}^{m}\left|w_{i}\right| \sum_{\substack{j=1, \mathbf{x}_{j} \in H_{i}^{ \pm} \backslash S\left( \pm \mathbf{x}_{i},\left(c_{1} / n\right)\right)}}^{m}\left|w_{j}\right|\left(\sin \theta_{i j}^{ \pm}\right)^{-5 / 2}
$$

where $\theta_{i j}^{ \pm}=\cos ^{-1}\left( \pm \mathbf{x}_{i} \cdot \mathbf{x}_{j}\right), \theta_{i j}^{ \pm} \in(0, \pi)$. Arguing as in Reimer [12], we define for $i=1, \ldots, m$

$$
g_{i}^{ \pm}(\theta):=\sum_{\substack{j=1, \mathbf{x}_{j} \in\left(H_{i}^{ \pm} \backslash S\left( \pm \mathbf{x}_{i}, c_{1} / n\right)\right) \cap S\left( \pm \mathbf{x}_{i}, \theta\right)}}^{m}\left|w_{j}\right|, \quad \theta \in\left[\frac{c_{1}}{n}, \frac{\pi}{2}\right]
$$

Thus $g_{i}^{ \pm}(\theta)$ is the contribution to the sum of the absolute values of the weights from the cubature points whose angular distance from $\pm \mathbf{x}_{i}$ lies in the interval $\left(\left(c_{1} / n\right), \theta\right]$ (except that the half open interval is replaced by the open interval in the case $\theta=\pi / 2$ and $g_{i}^{-}$). Note that $g_{i}^{\ddagger}$ vanishes at $c_{1} / n$, and (because it is monotone) that it is a function of bounded variation on $\left[c_{1} / n, \pi / 2\right]$. Define also

$$
f(\theta):=(\sin \theta)^{-5 / 2}, \quad \theta \in\left[\frac{c_{1}}{n}, \frac{\pi}{2}\right]
$$

and note that $f$ is a continuous function. This allows us to write the inner sum in (4.20) as a Riemann-Stieltjes integral (see [6, Chapter X]),

$$
\sum_{\substack{j=1, \mathbf{x}_{j} \in H_{i}^{ \pm} \backslash S\left( \pm \mathbf{x}_{i},\left(c_{1} / n\right)\right)}}^{m}\left|w_{j}\right|\left(\sin \theta_{i j}^{ \pm}\right)^{-5 / 2}=\int_{c_{1} / n}^{\pi / 2} f(\theta) d g_{i}^{ \pm}(\theta) .
$$


Integration by parts then yields

$$
\begin{aligned}
\int_{c_{1} / n}^{\pi / 2} f(\theta) d g_{i}^{ \pm}(\theta) & =f\left(\frac{\pi}{2}\right) g_{i}^{ \pm}\left(\frac{\pi}{2}\right)-f\left(\frac{c_{1}}{n}\right) g_{i}^{ \pm}\left(\frac{c_{1}}{n}\right)-\int_{c_{1} / n}^{\pi / 2} g_{i}^{ \pm}(\theta) d f(\theta) \\
& =g_{i}^{ \pm}\left(\frac{\pi}{2}\right)+\frac{5}{2} \int_{c_{1} / n}^{\pi / 2} g_{i}^{ \pm}(\theta)(\sin \theta)^{-7 / 2} \cos \theta d \theta
\end{aligned}
$$

On using (see Corollary 3)

$$
\left|g_{i}^{ \pm}(\theta)\right| \leqslant \sum_{\substack{j=1, \mathbf{x}_{j} \in S\left( \pm \mathbf{x}_{i}, \theta\right)}}^{m}\left|w_{j}\right| \leqslant 2 \pi c_{2}(\sin \theta)^{2}, \quad \theta \in\left[\frac{c_{1}}{n}, \frac{\pi}{2}\right],
$$

we finally obtain from $(4.20)$

$$
\begin{aligned}
\left|R^{ \pm}\right| & \leqslant c n^{-7 / 2}\left(\sum_{i=1}^{m}\left|w_{i}\right|\right) 2 \pi c_{2}\left(1+\frac{5}{2} \int_{c_{1} / n}^{\pi / 2} \frac{\cos \theta}{(\sin \theta)^{3 / 2}} d \theta\right) \\
& =2 \pi c_{2} c n^{-7 / 2}\left(\sum_{i=1}^{m}\left|w_{i}\right|\right)\left(1-5\left[(\sin \theta)^{-1 / 2}\right]_{c_{1} / n}^{\pi / 2}\right) \\
& \leqslant 2 \pi c_{2} c n^{-7 / 2}\left(4 \pi c_{2}\right)\left(-4+5\left(\sin \frac{c_{1}}{n}\right)^{-1 / 2}\right),
\end{aligned}
$$

where we have used (4.7) in the last step. Observing that $\sin (\theta) \geqslant(2 \theta) / \pi$ for $0 \leqslant \theta \leqslant \pi / 2$, we finally obtain

$$
\left|R^{ \pm}\right| \leqslant c n^{-7 / 2+1 / 2}=c n^{-3} .
$$

Putting this together with (4.19), (4.18) and (4.17) completes the proof.

REMARK 13. The key to the proof of the theorem is thus the representation of $K_{3 / 2}^{(n+1)}(\mathbf{x}, \mathbf{y})$ in Lemma 9 - a representation that might seem remarkable, in that for fixed $x \in S^{2}$ the critical first term, being a polynomial of degree $n$, is orthogonal to $K_{3 / 2}^{(n+1)}(\mathbf{x}, \cdot)$ in both $L_{2}$ and $H^{3 / 2}$.

\section{Proof of lemmas and a COROLlary in Section 4}

Proof of Lemma 2: The ideas of the proof of this lemma are taken from [14, Lemma 5.5.3], but as the statement and the proof are simplified here we include the proof.

The general idea is to cover the spherical cap $S(\mathbf{x}, r)$ with smaller spherical caps $S\left(\mathbf{y}, r_{0}\right)$ and to use the assumption (4.5) for these smaller caps. To achieve this we 
proceed in two steps: firstly, for $r_{1} \geqslant 0$ and $r_{2}=r_{1}+r_{0} \leqslant \pi$ we cover the spherical collar $S\left(\mathbf{x}, r_{1}, r_{2}\right)$, defined by

$$
S\left(\mathbf{x}, r_{1}, r_{2}\right):=\left\{\mathbf{y} \in S^{2} \mid r_{1}<\cos ^{-1}(\mathbf{x} \cdot \mathbf{y}) \leqslant r_{2}\right\}=S\left(\mathbf{x}, r_{2}\right) \backslash S\left(\mathbf{x}, r_{1}\right),
$$

by spherical caps $S\left(y, r_{0}\right)$ and derive a result analogous to (4.6) for such spherical collars. Then we cover our spherical cap $S(\mathbf{x}, r)$ with such spherical collars and the spherical cap $S\left(\mathbf{x}, r_{0}\right)$, and use (4.5) and the results for the spherical collars to prove (4.6).

PART 1. For fixed $\mathbf{x} \in S^{2}$, let $S\left(\mathbf{x}, r_{1}, r_{2}\right)$ be a spherical collar with $r_{1} \geqslant 0$ and $r_{2}=r_{1}+r_{0} \leqslant \pi$. The angle $\gamma:=\left(r_{1}+r_{2}\right) / 2$ is in $\left[\left(r_{0} / 2\right), \pi-\left(r_{0} / 2\right)\right]$, and we distinguish the two cases $\gamma \leqslant \pi / 2$ and $\gamma>\pi / 2$. We observe that

$$
S\left(\mathbf{x}, r_{1}, r_{2}\right)=S\left(-\mathbf{x}, \pi-r_{2}, \pi-r_{1}\right),
$$

and that

$$
\frac{\left(\pi-r_{2}\right)+\left(\pi-r_{1}\right)}{2}=\pi-\gamma
$$

If $\gamma>\pi / 2$ we consider $S\left(-\mathbf{x}, \pi-r_{2}, \pi-r_{1}\right)$; that is we regard $S\left(\mathbf{x}, r_{1}, r_{2}\right)$ as the spherical collar $S\left(-\mathbf{x}, \pi-r_{2}, \pi-r_{1}\right)$ with axis $-\mathbf{x}$, due to (5.1). Because of (5.2), we now obtain for $S\left(-\mathbf{x}, \pi-r_{2}, \pi-r_{1}\right)$ that $\left(\left(\pi-r_{2}\right)+\left(\pi-r_{1}\right)\right) / 2<\pi / 2$. Therefore we may restrict our discussion to the case of spherical collars $S\left(\mathbf{x}, r_{1}, r_{2}\right)$ for which $\gamma=\left(r_{1}+r_{2}\right) / 2 \leqslant \pi / 2$, and more precisely, $\gamma \in\left[r_{0} / 2, \pi / 2\right]$.

Let $\gamma \leqslant \pi / 2$ and consider the latitude $\ell_{\gamma}:=\left\{\mathbf{y} \in S^{2} \mid \mathbf{x} \cdot \mathbf{y}=\cos \gamma\right\}$, which is a circle of radius $\sin \gamma$. Choose $K:=\left\lceil 2 \pi r_{0}^{-1} \sin \gamma\right\rceil$ equally spaced points $\mathbf{y}_{1}, \ldots, \mathbf{y}_{K}$ on $\ell_{\gamma}$, from which it follows that the separation between $\mathbf{y}_{j}$ and $\mathbf{y}_{j+1}$ (and between $\mathbf{y}_{K}$ and $\mathbf{y}_{1}$ ) along $\ell_{\gamma}$ is at most $r_{0}$. We claim that the spherical caps $S\left(\mathbf{y}_{j}, r_{0}\right), j=1, \ldots, K$, cover our spherical collar $S\left(\mathrm{x}, r_{1}, r_{2}\right)$.

To show this we select an arbitrary point $\mathbf{y} \in S\left(\mathbf{x}, r_{1}, r_{2}\right)$, and show that it is contained in one of these caps. Let $\tilde{\mathbf{y}}$ denote the point of intersection (closest to $y$ ) of the great circle through $\mathbf{x}$ and $\mathbf{y}$ and the circle $\ell_{\gamma}$, and let $\mathrm{y}_{j}$ be the axis (of one of our spherical caps) which is closest to $\tilde{\mathbf{y}}$. Then an upper bound of the great circle (that is, geodesic) distance between $\mathrm{y}$ and $\mathrm{y}_{j}$ is given by the sum of the great circle distance between $\mathbf{y}$ and $\tilde{\mathbf{y}}$ and the distance between $\tilde{\mathbf{y}}$ and $\mathbf{y}_{j}$ along $\ell_{\gamma}$, that is the great circle distance between $y$ and $\mathbf{y}_{j}$ is bounded from above by $r_{0} / 2+r_{0} / 2=r_{0}$. Thus $\mathbf{y} \in S\left(\mathbf{y}_{j}, r_{0}\right)$.

It follows from this covering property and (4.5) that

$$
\begin{aligned}
\sum_{\substack{j=1, \mathbf{x}_{j} \in S\left(\mathbf{x}, r_{1}, r_{2}\right)}}^{m}\left|w_{j}\right| & \leqslant \sum_{\ell=1}^{K} c_{0}\left|S\left(\mathbf{y}_{\ell}, r_{0}\right)\right| \\
& \leqslant K c_{0}\left|S\left(\mathbf{y}_{1}, r_{0}\right)\right| \\
& \leqslant \pi r_{0}^{2} c_{0}\left(2 \pi r_{0}^{-1} \sin \gamma+1\right)
\end{aligned}
$$


where we have used $\left|S\left(\mathbf{y}_{1}, r_{0}\right)\right|=2 \pi\left(1-\cos r_{0}\right)=4 \pi\left(\sin \left(r_{0} / 2\right)\right)^{2} \leqslant \pi r_{0}^{2}$. Using $\sin r$ $\geqslant 2 r / \pi, 0 \leqslant r \leqslant \pi / 2$, we get the following lower bound for the surface area of the spherical collar $S\left(\mathbf{x}, r_{1}, r_{2}\right)$

$$
\begin{aligned}
\left|S\left(\mathbf{x}, r_{1}, r_{2}\right)\right| & =2 \pi\left(\cos r_{1}-\cos r_{2}\right) \\
& =4 \pi \sin \gamma \sin \left(r_{0} / 2\right) \\
& \geqslant 4 r_{0} \sin \gamma .
\end{aligned}
$$

Due to $\gamma \in\left[r_{0} / 2, \pi / 2\right]$, we have $\sin \gamma \geqslant \sin \left(r_{0} / 2\right)$, and thus also

$$
\left|S\left(\mathbf{x}, r_{1}, r_{2}\right)\right| \geqslant 4 r_{0} \sin \left(r_{0} / 2\right) \geqslant(4 / \pi) r_{0}^{2} .
$$

Combining (5.3), (5.4), and (5.5) yields

$$
\begin{aligned}
\sum_{\substack{j=1, \mathbf{x}_{j} \in S\left(\mathbf{x}, r_{1}, r_{2}\right)}}^{m}\left|w_{j}\right| & \leqslant \frac{\pi^{2}}{2} c_{0}\left|S\left(\mathbf{x}, r_{1}, r_{2}\right)\right|+\frac{\pi^{2}}{4} c_{0}\left|S\left(\mathbf{x}, r_{1}, r_{2}\right)\right| \\
& =\frac{3 \pi^{2}}{4} c_{0}\left|S\left(\mathbf{x}, r_{1}, r_{2}\right)\right| .
\end{aligned}
$$

Due to our initial observation, (5.6) is also valid for the case $\gamma>\pi / 2$.

PART 2. With the help of (5.6) and (4.5) we can now prove (4.6). For the spherical cap $S(\mathbf{x}, r)$, with $r \geqslant r_{0}$, there exists $k \in \mathbb{N}$ such that $k r_{0} \leqslant r<(k+1) r_{0}$. Then

$$
\begin{aligned}
\sum_{\substack{j=1, \mathbf{x}_{j} \in S(\mathbf{x}, r)}}^{m}\left|w_{j}\right| & \leqslant \sum_{\substack{j=1, \mathbf{x}_{j} \in S\left(\mathbf{x}, r_{0}\right)}}^{m}\left|w_{j}\right|+\sum_{i=.}^{k} \sum_{\substack{j=1, \mathbf{x}_{j} \in S\left(\mathbf{x}, r-i r_{0}, r-(i-1) r_{0}\right)}}^{m}\left|w_{j}\right| \\
& \leqslant c_{0}\left|S\left(\mathbf{x}, r_{0}\right)\right|+\sum_{i=1}^{k} \frac{3 \pi^{2} c_{0}}{4}\left|S\left(\mathbf{x}, r-i r_{0}, r-(i-1) r_{0}\right)\right| \\
& \leqslant c_{0}|S(\mathbf{x}, r)|+\frac{3 \pi^{2} c_{0}}{4}|S(\mathbf{x}, r)| \\
& =\left(1+\frac{3 \pi^{2}}{4}\right) c_{0}|S(\mathbf{x}, r)| .
\end{aligned}
$$

This completes the proof.

Proof of Lemma 9: Firstly, we rewrite the sum in the following way:

$$
\begin{aligned}
\frac{1}{2 \pi} \sum_{\ell=n+1}^{\infty}\left(\ell+\frac{1}{2}\right)^{-2} P_{\ell}(t)=\frac{2}{\pi} \sum_{\ell=n+1}^{\infty} \frac{1}{(2 \ell-1)(2 \ell+3)} P_{\ell}(t) \\
\quad-\frac{8}{\pi} \sum_{\ell=n+1}^{\infty} \frac{1}{(2 \ell-1)(2 \ell+1)^{2}(2 \ell+3)} P_{\ell}(t)
\end{aligned}
$$


Now we know (see Section 7) that

$$
\frac{2}{\pi} \sum_{\ell=0}^{\infty} \frac{1}{(2 \ell-1)(2 \ell+3)} P_{\ell}(t)=-\frac{\sqrt{2}}{2 \pi}(1-t)^{1 / 2}
$$

but in the first term on the right-hand side of $(5.7)$ the first $(n+1)$ terms of the series expansion (5.8) are missing. Therefore the first term in (5.7), denoted from now on by

$$
f^{(n+1)}(t):=\frac{2}{\pi} \sum_{\ell=n+1}^{\infty} \frac{1}{(2 \ell-1)(2 \ell+3)} P_{\ell}(t)
$$

is the error in the approximation of

$$
f(t):=-\frac{\sqrt{2}}{2 \pi}(1-t)^{1 / 2}
$$

by

$$
\frac{2}{\pi} \sum_{\ell=0}^{n} \frac{1}{(2 \ell-1)(2 \ell+3)} P_{\ell}(t)
$$

which is the $n$th partial sum of its Legendre series. In other words, $f^{(n+1)}$ is $f$ minus its $L_{2}([-1,1])$-orthogonal projection onto the space $\operatorname{Pol}_{n}([-1,1])$ of all polynomials in a single variable up to degree $n$ on $[-1,1]$.

More precisely, with the reproducing kernel $G_{n}$ of $\operatorname{Pol}_{n}([-1,1])$ (endowed with the norm $\|\cdot\|_{L_{2}([-1,1])}$,

$$
G_{n}(s, t):=\sum_{\ell=0}^{n} \frac{(2 \ell+1)}{2} P_{\ell}(s) P_{\ell}(t), \quad s, t \in[-1,1],
$$

the orthogonal projection $\mathcal{P}_{n}: L_{2}([-1,1]) \rightarrow \operatorname{Pol}_{n}([-1,1])$, defined by $\mathcal{P}_{n}^{2}=\mathcal{P}_{n}$ and $\left(\mathcal{P}_{n} g, h\right)_{L_{2}([-1,1])}=(g, h)_{L_{2}([-1,1])}$ for all $g \in L_{2}([-1,1])$ and for all $h \in \operatorname{Pol}_{n}([-1,1])$, is given by

$$
\mathcal{P}_{n} g:=\sum_{\ell=0}^{n} \frac{2 \ell+1}{2}\left(\int_{-1}^{1} g(s) P_{\ell}(s) d s\right) P_{\ell}(\cdot)=\int_{-1}^{1} g(s) G_{n}(s, \cdot) d s .
$$

In particular, $\mathcal{P}_{n} f=f-f^{(n+1)}$ and $\mathcal{P}_{n} 1=1$. Due to the Christoffel-Darboux formula (see $[19,(3.2 .3)]), G_{n}$ can be expressed in closed form as

$$
G_{n}(s, t)=\frac{(n+1)}{2} \frac{P_{n+1}(t) P_{n}(s)-P_{n}(t) P_{n+1}(s)}{t-s},
$$


and we obtain (using $\int_{-1}^{1} G_{n}(s, t) d s=\mathcal{P}_{n} 1=1$ )

$$
\begin{aligned}
f^{(n+1)}(t) & =f(t)-\mathcal{P}_{n} f(t) \\
& =\int_{-1}^{1}(f(t)-f(s)) G_{n}(s, t) d s \\
& =\frac{(n+1)}{2} \int_{-1}^{1} \frac{f(t)-f(s)}{t-s}\left(P_{n+1}(t) P_{n}(s)-P_{n}(t) P_{n+1}(s)\right) d s .
\end{aligned}
$$

Assuming from now on that $t \in[-1,1)$, we can rewrite the first factor in the integrand as follows:

$$
\frac{f(t)-f(s)}{t-s}=-\frac{\sqrt{2}}{2 \pi}\left(\frac{(1-s)^{1 / 2}}{(1-t)}+\frac{(1-t)^{3 / 2}-(1-s)^{3 / 2}}{(1-t)(t-s)}\right) .
$$

Thus

$$
f^{(n+1)}(t)=a_{n}(t)+b_{n}(t)
$$

where

$$
\begin{aligned}
& a_{n}(t):=\frac{(n+1)}{2(1-t)} \int_{-1}^{1} \frac{(-\sqrt{2})(1-s)^{1 / 2}}{2 \pi}\left(P_{n+1}(t) P_{n}(s)-P_{n}(t) P_{n+1}(s)\right) d s, \\
& b_{n}(t):=-\frac{\sqrt{2}}{2 \pi(1-t)} \int_{-1}^{1}\left((1-t)^{3 / 2}-(1-s)^{3 / 2}\right) G_{n}(s, t) d s .
\end{aligned}
$$

From the series expansion (5.8) we know that

$$
\int_{-1}^{1} \frac{(-\sqrt{2})(1-s)^{1 / 2}}{2 \pi} P_{\ell}(s) d s=\frac{4}{\pi(2 \ell-1)(2 \ell+1)(2 \ell+3)}, \quad \ell \geqslant 0 .
$$

Thus, we can compute $a_{n}(t)$ exactly:

$$
\begin{aligned}
a_{n}(t) & =\frac{2}{\pi} \frac{(n+1)}{(1-t)}\left(\frac{P_{n+1}(t)}{(2 n-1)(2 n+1)(2 n+3)}-\frac{P_{n}(t)}{(2 n+1)(2 n+3)(2 n+5)}\right) \\
& =\frac{2}{\pi} \frac{(n+1)}{(1-t)}\left(\frac{P_{n+1}(t)-P_{n}(t)}{(2 n-1)(2 n+1)(2 n+3)}+\frac{6 P_{n}(t)}{(2 n-1)(2 n+1)(2 n+3)(2 n+5)}\right) .
\end{aligned}
$$

Using $P_{n}(t)-P_{n+1}(t)=(1-t) P_{n}^{(1,0)}(t)$, which follows easily from Rodrigues' formula (see $[19,(4.3 .1)])$, we get

$$
\begin{aligned}
a_{n}(t)=-\frac{2(n+1)}{\pi(2 n-1)(2 n+1)(2 n+3)} P_{n}^{(1,0)}(t) \\
\quad+\frac{12(n+1)}{\pi(2 n-1)(2 n+1)(2 n+3)(2 n+5)} \frac{P_{n}(t)}{(1-t)} .
\end{aligned}
$$


Now, we turn to $b_{n}(t)$. It follows from (5.12) (compare (5.10)) that

$$
b_{n}(t)=-\frac{\sqrt{2}}{2 \pi(1-t)}\left(g(t)-\mathcal{P}_{n} g(t)\right)
$$

where

$$
g(t)=(1-t)^{3 / 2}
$$

But we know that (see Section 7)

$$
(1-t)^{3 / 2}=\sum_{\ell=0}^{\infty} \frac{36 \sqrt{2}}{(2 \ell-3)(2 \ell-1)(2 \ell+3)(2 \ell+5)} P_{\ell}(t), \quad t \in[-1,1] .
$$

In analogy to (5.10), we can therefore write $b_{n}(t)$ as

$$
b_{n}(t)=-\frac{36}{\pi(1-t)} \sum_{\ell=n+1}^{\infty} \frac{1}{(2 \ell-3)(2 \ell-1)(2 \ell+3)(2 \ell+5)} P_{\ell}(t)
$$

The identities (5.7), (5.9), (5.11), (5.13), and (5.15) together imply (4.12) in Lemma 9.

Proof of Corollary 10: The estimate (4.13) follows from (4.11), (4.12), and the estimate

$$
(1-\cos \theta)^{-1}=(\sin \theta)^{-2}(1+\cos \theta) \leqslant 2(\sin \theta)^{-2}
$$

and from $[19,(7.3 .8)]$,

$$
\left|P_{\ell}(\cos \theta)\right|(\sin \theta)^{1 / 2}<\left(\frac{2}{\pi}\right)^{1 / 2} \ell^{-1 / 2}, \quad 0 \leqslant \theta \leqslant \pi .
$$

It follows from (4.11), (4.12), and (4.13) that for fixed $\theta \in(0, \pi)$

$$
K_{3 / 2}^{(n+1)}(\mathbf{x}, \mathbf{y})=-\frac{2}{\pi} \frac{(n+1)}{(2 n-1)(2 n+1)(2 n+3)} P_{n}^{(1,0)}(\cos \theta)+O\left(n^{-7 / 2}\right) .
$$

Now it is known that for $0<\theta<\pi$

$$
P_{n}^{(1,0)}(\cos \theta)=\pi^{-1 / 2} n^{-1 / 2}\left(\sin \frac{\theta}{2}\right)^{-3 / 2}\left(\cos \frac{\theta}{2}\right)^{-1 / 2} \cos \left((n+1) \theta-\frac{3}{4} \pi\right)+O\left(n^{-3 / 2}\right),
$$

which is a special case of Darboux's asymptotic formula (see [19, Theorem 8.21.8]), thus (4.14) follows with

$$
D_{n}(\theta)=-\frac{1}{4 \pi^{3 / 2}}\left(\sin \frac{\theta}{2}\right)^{-3 / 2}\left(\cos \frac{\theta}{2}\right)^{-1 / 2} \cos \left((n+1) \theta-\frac{3}{4} \pi\right) .
$$

Proof of Lemma 12: As $P_{n}^{(1,0)}\left(\mathbf{x}_{i} \cdot \mathbf{x}\right) \in \mathbb{P}_{n}$ for all $i \in\{1, \ldots, m\}$, the cubature rule $Q_{m}$ integrates it exactly. That is,

$$
\begin{aligned}
\sum_{i=1}^{m} w_{i} \sum_{j=1}^{m} w_{j} P_{n}^{(1,0)}\left(\mathbf{x}_{i} \cdot \mathbf{x}_{j}\right) & =\sum_{i=1}^{m} w_{i} \int_{S^{2}} P_{n}^{(1,0)}\left(\mathbf{x}_{i} \cdot \mathbf{y}\right) d \omega(\mathbf{y}) \\
& =\sum_{i=1}^{m} w_{i} \frac{4 \pi}{(n+1)}=\frac{16 \pi^{2}}{(n+1)}
\end{aligned}
$$


where we have used (4.1) and

$$
\int_{-1}^{1} P_{n}^{(1,0)}(s) d s=\frac{2}{(n+1)},
$$

which can be easily proved with the help of Rodrigues' formula (see [19, (4.3.1)]).

\section{EXAMPLES}

In this section we discuss examples of cubature rules that satisfy both the exactness property (1.4) and the regularity property (R) of Theorem 6 and Corollary 8 . For sequences of such rules, the $O\left(n^{-3 / 2}\right)$ estimate for the worst-case error in $H^{3 / 2}$ therefore applies, with a constant $c$ independent of the sequence in the case of positive weight rules.

6.1. Product RUles. The most popular cubature rules over $S^{2}$ are product rules. Writing the integrand in (1.2) as

$$
f(\sin \theta \cos \phi, \sin \theta \sin \phi, \cos \theta)=F(\cos \theta, \phi), \quad \text { for } \theta \in[0, \pi], \phi \in[0,2 \pi),
$$

the integral (1.2) may be written as

$$
I f=\int_{0}^{2 \pi} \int_{-1}^{1} F(t, \phi) d t d \phi .
$$

A product rule is then a rule of the form

$$
Q_{m} f:=\frac{2 \pi}{n+1} \sum_{j=0}^{n} \sum_{\ell=1}^{n^{\prime}} \mu_{\ell} F\left(t_{\ell}, \frac{j 2 \pi}{n+1}\right),
$$

which is just the product of two 1-dimensional rules:

$$
\frac{2 \pi}{n+1} \sum_{j=0}^{n} g\left(\frac{j 2 \pi}{n+1}\right) \approx \int_{0}^{2 \pi} g(\phi) d \phi,
$$

a rule which integrates exactly all trigonometric polynomials of degree $\leqslant n$; and

$$
\sum_{\ell=1}^{n^{\prime}} \mu_{\ell} h\left(t_{\ell}\right) \approx \int_{-1}^{1} h(t) d t,
$$

a rule which we shall require to have positive weights $\mu_{\ell}$ and to integrate exactly all algebraic polynomials of degree $\leqslant n$. It is easily seen that every such product rule satisfies (1.4). Because they also have positive weights, a sequence $\left(Q_{m(n)}\right)$ of such rules also has the property (R), as we remarked after Theorem 6 . 
There are many possible choices for the rule (6.2). For example, the rule could be a Gauss rule [2], in which case $n^{\prime}=\lceil(n+1) / 2]$, or a Clenshaw-Curtis rule [2], in which case $n^{\prime}=n+1$. The total number of points of the cubature rule is $m=(n+1) n^{\prime}$, if we allow the possibility of cubature points coinciding. The value of $m$ can be reduced to $m=(n+1)\left(n^{\prime}-2\right)+2$ if (as in the Clenshaw-Curtis rule or the Lobatto rule [2]) the set of quadrature points $\left\{t_{\ell}\right\}$ in (6.2) includes the two endpoints.

We observe that in the examples above $m$ is exactly of the order $n^{2}$, thus the rate of convergence of $E_{3 / 2}\left(Q_{m(n)}\right)$ when expressed in terms of $m$ is $O\left(m^{-3 / 4}\right)$, which is believed to be optimal.

6.2. SPherical $n$-DEsigns. A point set $\left\{x_{1}, \ldots, x_{m}\right\} \subset S^{2}$ is called a spherical $n$-design on $S^{2}$ if the cubature rule

$$
Q_{m} f:=\frac{4 \pi}{m} \sum_{j=1}^{m} f\left(\mathbf{x}_{j}\right)
$$

is exact for all polynomials of degree $\leqslant n$. That is to say, if the weights in (1.3) are given by $w_{j}=4 \pi / m$ and (1.4) is satisfied. As noted already, for a sequence of positiveweight rules such as this the regularity property $(R)$ holds automatically, and therefore Theorem 6 applies.

The concept of spherical designs was introduced in [3], and in [13] the existence of spherical $n$-designs for any $n$ and sufficiently large $m$ was proved. For practical purposes it is, of course, desirable to have spherical $n$-designs with a small number of points $m$.

For the minimal number $M(n)$ of points such that a spherical $n$-design with $m=M(n)$ points exists, the lower bound

$$
M(n) \geqslant c n^{2}
$$

is known (see $[1,3])$.

In [5] for $n=2 q-1 \geqslant 1$, spherical $n$-designs with $O\left(n^{3}\right)$ points were constructed as product rules, but these are not serious contenders as cubature rules, as they use too many points. Whether spherical $n$-designs with $O\left(n^{2}\right)$ points exist for arbitrarily large $n$ seems to be as yet unknown. (Rabau and Bajnok [10, Conjecture 4.4], conjecture that the minimum number of points of a spherical $n$-design on $S^{2}$ is of order $O\left(n^{3}\right)$, but in [5] Korevaar and Meyers conjecture that the minimum number of points is $O\left(n^{2}\right)$; we support the second conjecture.) Finally, in [1] it is shown that the minimum number of points $M^{\prime}(n)$ such that for all $m \geqslant M^{\prime}(n)$ a spherical $n$-design of $m$ points exists, has the upper bound

$$
M^{\prime}(n) \leqslant c n^{5.5} \text {. }
$$

6.3. RULES OF SOBOLEV TYPE. Sobolev [17] introduced the concept of cubature rules that are invariant under a finite symmetry group of the sphere, based on the observation 
that if $a$ is the lowest degree of the non-trivial invariant polynomials under the particular group, then all spherical polynomials of degree less than $a$ are integrated exactly. Lebedev and co-workers $[7,8]$ have constructed many rules on the sphere by exploiting the Sobolev theory for the particular case of the octahedral group with inversion, with values of $n$ in equation (1.4) as high as $n=131$ (see [8]). The weights are often positive. As long as this is the case (given that positivity of the weights is not guaranteed) the conclusion of Theorem 6 applies. The number of points $m$ is always $O\left(n^{2}\right)$, and indeed is smaller than the number of points for product rules that are exact for polynomials up to degree $n$.

6.4. Extremal systems. An extremal system (see [11]) on $S^{2}$ is a set of $(n+1)^{2}$ distinct points $\left\{\mathbf{x}_{j}\right\}$ for which the determinant of the interpolation matrix $\left\{Y_{\ell k}\left(\mathbf{x}_{j}\right)\right\}_{0 \leqslant \ell \leqslant n, k=1, \ldots, 2 \ell+1}^{j=1, \ldots,(n+1)^{2}}$ takes its maximal value. In a recent study [15] of interpolatory cubature on $S^{2}$ with respect to extremal systems (which by definition has the property (1.4)) it was found numerically that the resulting weights are all positive for all degrees $n$ up to at least $n=50$.

Of course, the conclusions of Theorem 6 hold as long as the weights are positive, but an alternative condition on the absolute values of the weights would also be adequate, because of the good geometrical distribution of extremal points. Specifically, the following property of extremal systems is known:

TheOREM 14. (See [11].) For $n \geqslant 1$, let $\mathrm{x}_{1}, \ldots, \mathrm{x}_{(n+1)^{2}}$ be an extremal system on $S^{2}$. Then

$$
\cos ^{-1}\left(\mathbf{x}_{j} \cdot \mathbf{x}_{k}\right) \geqslant \frac{\pi}{2 n} \quad \text { for } j \neq k .
$$

Because of this result, the property $(R)$ holds if the ratio of the weights to the average weight is bounded uniformly in $n$.

PROPOSITION 15. For $n \geqslant 1$ and $m(n)$ satisfying $a n^{2} \leqslant m(n) \leqslant b n^{2}$ for some $a, b>0$, independent of $n$, let $\mathbf{x}_{1}, \ldots, \mathbf{x}_{m(n)}$ be a set of distinct points on $S^{2}$ that satisfy (6.3). Let $\left(Q_{m(n)}\right)$ be a sequence of cubature rules $Q_{m(n)}$, given by (1.3), with the points $\mathbf{x}_{1}, \ldots, \mathbf{x}_{m(n)}$, and with the corresponding weights satisfying

$$
\left|w_{j}\right| \leqslant c_{3} \frac{4 \pi}{m(n)}, \quad j=1, \ldots, m(n),
$$

for some constant $c_{3}>0$, independent of $n$. Then the property $(R)$ holds with $c_{0}=\left(32 c_{3}\right) / a$ and $c_{1}=\pi / 4$.

Proof of Proposition 15: Because of the bound on the weights, for all $\mathbf{x} \in S^{2}$ we have

$$
\sum_{\substack{j=1,1 \\ \mathbf{x}_{j} \in S(\mathbf{x}, \pi /(4 n))}}^{m(n)}\left|w_{j}\right| \leqslant \frac{4 \pi c_{3}}{m(n)} \operatorname{card}\left\{j \in\{1, \ldots, m(n)\} \mid \mathbf{x}_{j} \in S\left(\mathbf{x}, \frac{\pi}{4 n}\right)\right\} .
$$


Now because of the condition (6.3), at most two of the cubature points can lie in $S(\mathbf{x}, \pi /(4 n)$ ) (this happens if and only if the two points lie at opposite ends of a 'diameter' of the spherical cap). Thus

$$
\sum_{\substack{j=1, \mathbf{x}} \in S\left(\mathbf{x}, \frac{\pi}{4 n}\right)}^{m(n)}\left|w_{j}\right| \leqslant \frac{8 \pi c_{3}}{m(n)} \leqslant \frac{8 \pi c_{3}}{a n^{2}} \leqslant \frac{32 c_{3}}{a}\left|S\left(\mathbf{x}, \frac{\pi}{4 n}\right)\right|,
$$

where in the last step we used the easily found bound

$$
\frac{|S(\mathbf{x}, r)|}{\pi r^{2}} \geqslant \frac{4}{\pi^{2}}, \quad 0<r \leqslant \pi
$$

with $r=\pi /(4 n)$. Thus the property (R) holds, with $c_{1}=\pi / 4$ and $c_{0}=\left(32 c_{3}\right) / a$.

\section{SOME SERIES EXPANSIONS IN LEGENDRE POLYNOMIALS}

In this section we sketch derivations of the series expansions (5.8) and (5.14). The functions $(1-t)^{1 / 2}$ and $(1-t)^{3 / 2}$ are both in $L_{2}([-1,1])$ and have therefore a series expansion (in the Lebesgue sense) with respect to the $L_{2}([-1,1])$-complete orthonormal system $\left\{((2 n+1) / 2)^{1 / 2} P_{n}\right\}_{n \in N_{0}}$. Thus

$$
(1-t)^{1 / 2}=\sum_{\ell=0}^{\infty}\left(\int_{-1}^{1}(1-s)^{1 / 2} P_{\ell}(s) d s\right) \frac{(2 \ell+1)}{2} P_{\ell}(t)
$$

and analogously for $(1-t)^{3 / 2}$. In order to compute the integrals, we observe that

$$
\int_{-1}^{1}(1-s)^{\alpha} P_{\ell}(s) d s
$$

is well-defined for $\alpha>-1$. Using the Legendre polynomial property that (see $[4,(3.2 .19)])$

$$
(2 \ell+1) P_{\ell}(t)=P_{\ell+1}^{\prime}(t)-P_{\ell-1}^{\prime}(t), \quad \ell \in \mathbb{N},
$$

we derive by partial integration, for $\alpha>0$ and $\ell \geqslant 1$, the relation

$$
\begin{aligned}
\int_{-1}^{1}(1-s)^{\alpha} & P_{\ell}(s) d s \\
& =\frac{\alpha}{(2 \ell+1)}\left(\int_{-1}^{1}(1-s)^{\alpha-1} P_{\ell+1}(s) d s-\int_{-1}^{1}(1-s)^{\alpha-1} P_{\ell-1}(s) d s\right) .
\end{aligned}
$$

It follows from (7.2) that the integrals (7.1) with $\alpha=1 / 2$, and in turn the integrals with $\alpha=3 / 2$, can be evaluated once the integrals (7.1) with $\alpha=-1 / 2$ are known. 
The key result we need is therefore

$$
\frac{1}{\sqrt{2}} \int_{-1}^{1}(1-s)^{-1 / 2} P_{\ell}(s) d s=\frac{2}{(2 \ell+1)}, \quad \ell \in \mathbb{N}_{0} .
$$

We sketch briefly how this can be proved. The basic idea is to use (see, for example $[4$, Lemma 3.2.4]) the generating function of the Legendre polynomials,

$$
\left(1+h^{2}-2 h t\right)^{-1 / 2}=\sum_{k=0}^{\infty} h^{k} P_{k}(t), \quad t \in[-1,1], h \in(-1,1) .
$$

For each fixed $h \in(-1,1)$ the function on the left-hand side of (7.4) is infinitely often differentiable on $[-1,1]$ and its series expansion on the right-hand side converges uniformly for $t \in[-1,1]$. Taking the limit $h \rightarrow 1$ we get for the left-hand side of (7.4)

$$
\lim _{h \rightarrow 1}\left(1+h^{2}-2 h t\right)^{-1 / 2}=\frac{1}{\sqrt{2}}(1-t)^{-1 / 2}, \quad t \in[-1,1) .
$$

For fixed $\ell \geqslant 0$ we define now $f_{h}^{(\ell)}:[-1,1] \rightarrow \mathbb{R}$ for $h \in\left[h_{0}, 1\right), h_{0}>0$, by

$$
f_{h}^{(\ell)}(t)=\frac{P_{\ell}(t)}{\left(1+h^{2}-2 h t\right)^{1 / 2}} \text {. }
$$

Obviously

$$
\lim _{h \rightarrow 1} f_{h}^{(\ell)}(t)=\frac{P_{\ell}(t)}{\sqrt{2}(1-t)^{1 / 2}}=: f^{(\ell)}(t), \quad t \in[-1,1),
$$

and the estimate

$$
1+h^{2}-2 h t=(1-h t)^{2}+h^{2}(1-t)(1+t) \geqslant h_{0}^{2}(1-t)(1+t)
$$

for $t \in[-1,1], h \in\left[h_{0}, 1\right)$, implies for $t \in(-1,1)$

$$
\left|f_{h}^{(\ell)}(t)\right| \leqslant \frac{\left|P_{\ell}(t)\right|}{h_{0}(1-t)^{1 / 2}(1+t)^{1 / 2}}=: g^{(\ell)}(t) .
$$

The function $g^{(\ell)}$ is in $L_{1}([-1,1])$ and independent of $h$. Thus the dominated convergence theorem (see [6, Chapter VI, Theorem 5.8]) implies that $\lim _{h \rightarrow 1} f_{h}^{(\ell)}=f^{(\ell)}$ in the Lebesgue sense, and in particular

$$
\lim _{h \rightarrow 1} \int_{-1}^{1} f_{h}^{(\ell)}(s) d s=\int_{-1}^{1} f^{(\ell)}(s) d s=\frac{1}{\sqrt{2}} \int_{-1}^{1} \frac{P_{\ell}(s)}{(1-s)^{1 / 2}} d s .
$$

Now for $h \in\left[h_{0}, 1\right)$ fixed it is clear that (7.4) converges uniformly, thus

$$
\int_{-1}^{1} f_{h}^{(\ell)}(s) d s=\sum_{k=0}^{\infty} h^{k} \int_{-1}^{1} P_{k}(s) P_{\ell}(s) d s=h^{\ell} \frac{2}{(2 \ell+1)} .
$$


This proves (7.3).

Application of (7.2), starting with $\alpha=1 / 2$ and using (7.3), yields in the $L_{2}([-1,1])$ sense

$$
\begin{aligned}
& (1-t)^{1 / 2}=-\sum_{\ell=0}^{\infty} \frac{2 \sqrt{2}}{(2 \ell-1)(2 \ell+3)} P_{\ell}(t) \\
& (1-t)^{3 / 2}=\sum_{\ell=0}^{\infty} \frac{36 \sqrt{2}}{(2 \ell-3)(2 \ell-1)(2 \ell+3)(2 \ell+5)} P_{\ell}(t) .
\end{aligned}
$$

Both series also converge uniformly on $[-1,1]$, and therefore (7.5) and (7.6) are valid pointwise.

\section{REFERENCES}

[1] B. Bajnok, 'Construction of designs on the 2-sphere', European J. Combin. 12 (1991), 377-382.

[2] P.J. Davis and P. Rabinowitz, Methods of numerical integration (Academic Press, Orlando, FL, 1984).

[3] P. Delsarte, J.M. Goethals and J.J. Seidel, 'Spherical codes and designs', Geom. Dedicata 6 (1977), 363-388.

[4] W. Freeden, T. Gervens and M. Schreiner, Constructive approximation on the sphere with applications to geomathematics, Numerical Mathematics and Scientific Computation (Oxford University Press, New York, 1998).

[5] J. Korevaar and J.L.H. Meyers, 'Spherical Faraday cage for the case of equal point charges and Chebyshev-type quadrature on the sphere', Integral Transform Spec. Funct. 1 (1993), 105-117.

[6] S. Lang, Real and functional analysis, (3rd edition) (Springer-Verlag, New York, 1993).

[7] V.I. Lebedev, 'Quadratures on a sphere', (in Russian), Z. Vyčchisl. Mat. i mat. Fiz. 16 (1976), 293-306.

[8] V.I. Lebedev and D.N. Laikov, 'A quadrature formula for the sphere of 131st algebraic order of accuracy', (in Russian), Dokl. Akad. Nauk 366 (1999), 741-745.

[9] C. Müller, Spherical harmonics, Lecture Notes in Mathematics 17 (Springer-Verlag, Berlin, 1966).

[10] P. Rabau and B. Bajnok, 'Bounds for the number of nodes in Chebyshev type quadrature formulas', J. Approx. Theory 67 (1991), 199-214.

[11] M. Reimer, Constructive theory of multivariate functions (B.I. Wissenschaftsverlag, Mannheim, Zürich, 1990).

[12] M. Reimer, 'Hyperinterpolation on the sphere at the minimal projection order', J. Approx. Theory 104 (2000), 272-286.

[13] P.D. Seymour and T. Zaslavsky, 'Averaging sets: a generalization of mean values and spherical designs', Adv. in Math. 52 (1984), 213-240.

[14] I.H. Sloan and R.S. Womersley, 'Constructive polynomial approximation on the sphere', J. Approx. Theory 103 (2000), 91-118. 
[15] I.H. Sloan and R.S. Womersley, 'Extremal systems of points and numerical integration on the sphere', Adv. Comput. Math. 21 (2004), 107-125.

[16] I.H. Sloan and H. Woźniakowski, 'When are quasi-Monte Carlo algorithms efficient for high dimensional integrals?', J. Complexity 14 (1998), 1-33.

[17] S.L. Sobolev, 'Cubature formulae on the sphere invariant under finite groups of rotations', Soviet Mathematics 3 (1962), 1307-1310.

[18] A.H. Stroud, Approximate calculation of multiple integrals (Prentice Hall, Englewood Cliffs, N.J., 1971).

[19] G. Szegö, Orthogonal polynomials (4th edition), American Mathematical Society Colloquium Publications 23 (American Mathematical Society, Providence, 1975).

School of Mathematics

The University of New South Wales

Sydney NSW 2052

Australia

e-mail: k.hesse@unsw.edu.au
School of Mathematics

The University of New South Wales

Sydney NSW 2052

Australia

e-mail: i.sloan@unsw.edu.au 